OPEN ACCESS

Edited by: Ildikó Rácz,

Universitätsklinikum Bonn, Germany

Reviewed by:

Livio Luongo,

Università degli Studi della Campania

"Luigi Vanvitelli" Naples, Italy

Bogna Ignatowska-Jankowska,

Okinawa Institute of Science and

Technology Graduate University,

Japan

*Correspondence:

Lucyna Antkiewicz-Michaluk antkiew@if-pan.krakow.pl Katarzyna Starowicz starow@if-pan.krakow.pl

Received: 28 September 2018 Accepted: 03 December 2018 Published: 20 December 2018

Citation:

Mlost J, Wąsik A, Michaluk JT, Antkiewicz-Michaluk $L$ and Starowicz K (2018) Changes in Monoaminergic Neurotransmission in an Animal Model of Osteoarthritis:

The Role of Endocannabinoid Signaling.

Front. Mol. Neurosci. 11:466. doi: 10.3389/fnmol.2018.00466

\section{Changes in Monoaminergic Neurotransmission in an Animal Model of Osteoarthritis: The Role of Endocannabinoid Signaling}

\author{
Jakub Mlost, Agnieszka Wąsik, Jerzy Tadeusz Michaluk, Lucyna Antkiewicz-Michaluk* \\ and Katarzyna Starowicz*
}

Department of Neurochemistry, Institute of Pharmacology, Polish Academy of Sciences, Kraków, Poland

Chronic pain is a main symptom of osteoarthritis (OA). Moreover, a high percentage of OA patients suffer from mental health problems. The endocannabinoid (EC) system has attracted attention as an emerging drug target for pain treatment together with its activity on the mesolimbic reward system. Understanding the circuits that govern the reward of pain relief is crucial for the search for effective analgesics. Therefore, we investigated the role of the EC system on dopamine (DA) and noradrenaline (NA) in an animal model of OA-related chronic pain. OA rats exhibited significant decreases in DA metabolism in the nucleus accumbens (NAc), striatum (STR) and hippocampus (HC). NA metabolism was also significantly decreased by chronic pain in OA rats; however, this disruption was limited to the frontal cortex (FCx) and HC. URB597 (an inhibitor of EC metabolism) treatment completely reversed the decreased DA metabolism, especially in the brain reward system and the HC. Furthermore, administration of URB597 normalized the impairment of NA activity in the $\mathrm{HC}$ but potentiated the decreased NA levels in the FCx. Our results demonstrated that chronic pain in OA rats was reflected by the inhibition of mesolimbic and mesocortical dopaminergic transmission, and may indicate the pro-pain role of NA in the FCx. The data provide understanding about changes in neurotransmission in chronic pain states and may explain the clinical improvement in perceived life quality following cannabinoid treatment. Additional mechanistic studies in preclinical models examining the intersection between chronic pain and reward circuits may offer new approaches for improving pain therapy. Keywords: osteoarthritis, chronic pain, catecholamines metabolism, brain structures, reward system,
hypodopaminergia, endocannabinoid system

\section{INTRODUCTION}

According to the International Association for the Study of Pain (IASP), pain is defined as a complicated phenomenon compromising an unpleasant sensory and emotional experience associated with actual or potential tissue damage. Pain is a crucial evolutionary mechanism designed for the survival of organisms. However, long-lasting noxious stimulation is no longer serving its evolutionary role and turning into an unbearable experience.

Pain chronification involves structural and functional changes in the central nervous system (Apkarian et al., 2013; Mansour et al., 2013). Accumulating evidence has demonstrated that structures engaged in reward circuits are also involved in the transition from acute to chronic pain (Baliki et al., 2012; Vachon-Presseau et al., 2016). Recent work from Martikainen et al. (2015) 
showed a significant decrease in dopamine (DA) signaling in the ventral tegmental area, in chronic pain patients, and this decrease was negatively correlated with pain sensitivity.

Moreover, there is a high comorbidity between chronic pain and mental health problems related to dopaminergic signaling dysfunction. For instance, Nazarinasab reported in 2017, that more than half of osteoarthritic patients suffered from mental health problems, such as obsessive-compulsive disorder, depression or anxiety (Leite et al., 2011; Nazarinasab et al., 2017). Additionally, the risk of suicide is considerably high among chronic pain patients (Hassett et al., 2014). Strikingly, even a higher number (up to $80 \%$ ) of major depression or Parkinson's disease patients report comorbid pain conditions (Leuchter et al., 2010; Skogar and Lökk, 2016; Young Blood et al., 2016). Further research showed that Parkinson's disease patients report higher pain ratings while off their treatment (levodopa, orally bioavailable precursor of DA; Nebe and Ebersbach, 2009).

Current treatment of chronic pain is based on opioid drugs that exertpronounced antinociceptive effects and have a strong rewarding effect that often leads to addiction and a phenomenon called opioid-induced hyperalgesia. Alternatively, targeting the endocannabinoid (EC) system could become a novel treatment strategy for chronic pain patients. EC consists of ECs, enzymes responsible for their metabolism and receptors, $\mathrm{CB} 1$ and $\mathrm{CB} 2$, both of which are highly implicated in pain and reward processing. Targeting enzymes involved in the degradation of ECs seems to be a promising treatment strategy as ECs are produced "on demand" and therefore, inhibition of degradative enzymes (such as fatty acid amide hydrolase, FAAH) should be devoid of unwanted side effects as the increase of EC levels should be limited specifically to disease-affected tissues.

The aim of our research was to evaluate the DA and noradrenaline (NA) concentrations and the rate of their metabolism in mesolimbic and mesocortical brain structures in response to osteoarthritis (OA)-related pain, and to monitor the effect of EC system activation.

\section{MATERIALS AND METHODS}

\section{Animals}

Male Wistar rats (Charles River, Hamburg, Germany), initially weighing 225-250 g, were used for all experiments. Animals were housed five per cage under a standard $12 \mathrm{~h} / 12 \mathrm{~h}$ light/dark cycle (lights on at 06:00 h) with food and water available ad libitum. This study was carried out in accordance with the principles of the Basel Declaration and the recommendations of the IASP and 3R policy [IASP Guidelines for the Use of Animals in Research; International Association for the Study of Pain (IASP), 2018]. The protocol was approved by the Local Ethics Committee of the Institute of Pharmacology (Cracow, Poland, approval number 125/2018). Care was taken to implement the "3 Rs" rule (replacement, reduction and refinement) to reduce the number of animals used and their suffering during the experiments.

\section{OA Induction}

The rats were deeply anesthetized with $5 \%$ isoflurane (Forane ${ }^{\circledR}$, Baxter Healthcare Corporation, Deerfield, IL, USA) in $100 \%$
$\mathrm{O}_{2}(3 \mathrm{~L} / \mathrm{min})$. The skin covering the right knee joint was shaved and swabbed with $100 \%$ ethanol. A 27 -gauge needle was introduced into the joint cavity through the patellar ligament, and $50 \mu \mathrm{l}$ containing $1 \mathrm{mg}$ of sodium monoiodoacetate (MIA) in $0.9 \%$ saline was injected intra-articular to induce OA-like lesions.

\section{Drugs}

MIA, dimethyl sulfoxide (DMSO), and Kolliphor EL were obtained from Sigma-Aldrich (Poznan, Poland). URB-597 was obtained from Tocris Bioscience (Bristol, UK); URB597 was dissolved in 5\% DMSO, 5\% ethanol, 5\% Kolliphor EL and 85\% saline at a $1 \mathrm{mg} / \mathrm{ml}$ concentration. MIA was dissolved in $50 \mu \mathrm{l}$ of $0.9 \%$ saline.

\section{Ex vivo Biochemical Studies}

At day 28 post MIA injection, rats were treated with $1 \mathrm{mg} / \mathrm{kg}$ of URB597 i.p. and $1 \mathrm{~h}$ later sacrificed by decapitation. Brains were rapidly removed and dissected on an ice-cold glass plate, and the following structures were isolated: striatum (STR), nucleus accumbens (NAc), hippocampus (HC) and frontal cortex (FCx). The obtained tissue was frozen on solid $\mathrm{CO}_{2}\left(-70^{\circ} \mathrm{C}\right)$ and stored until used for biochemical assays. DA and its final metabolite homovanillic acid (HVA), $\mathrm{NA}$ and its extracellular metabolite normetanephrine (NM), were assayed by high-performance liquid chromatography (HPLC) with electrochemical detection. The tissue samples were weighed and homogenized in ice-cold $0.1 \mathrm{M}$ perchloroacetic acid containing $0.05 \mathrm{mM}$ ascorbic acid. After centrifugation $(10,000 \times g, 5 \mathrm{~min})$, the supernatants were filtered through RC $58 \quad 0.2 \mu \mathrm{m}$ cellulose membranes (Bioanalytical Systems, West Lafayette, IN, USA). The mobile phase consisted of $0.05 \mathrm{M}$ citrate-phosphate buffer ( $\mathrm{pH} 3.5), 0.1 \mathrm{mM}$ EDTA, $1 \mathrm{mM}$ sodium octyl sulfonate and $3.5 \%$ methanol. The HP 1050 chromatograph (Hewlett-Packard, Golden, CO, USA) was equipped with a C18 column. The flow rate was maintained at $1 \mathrm{ml} / \mathrm{min}$. DA, NA and their metabolites were quantified by peak height compared with standards run on the day of the analysis. Each group comprised 5-8 animals.

\section{Statistical Analysis}

One-way analysis of variance (ANOVA) was used to analyze the results from the neurochemical studies. Differences between the control and experimental groups were assessed using Duncan's post hoc tests. The data were considered statistically significant when $P<0.05$.

The total metabolism rate for DA was assessed from the ratio of the final DA metabolite HVA concentration to DA concentration and expressed as the catabolic rate index $[\mathrm{HVA}] /[\mathrm{DA}] \times 100$; analogously, the rate of NA metabolism was expressed as the ratio of the extraneuronal NA metabolite concentration $[\mathrm{NM}]$ to $[\mathrm{NA}] \times 100$. The indices were calculated using concentrations from individual tissue samples (Antkiewicz-Michaluk et al., 2001). 

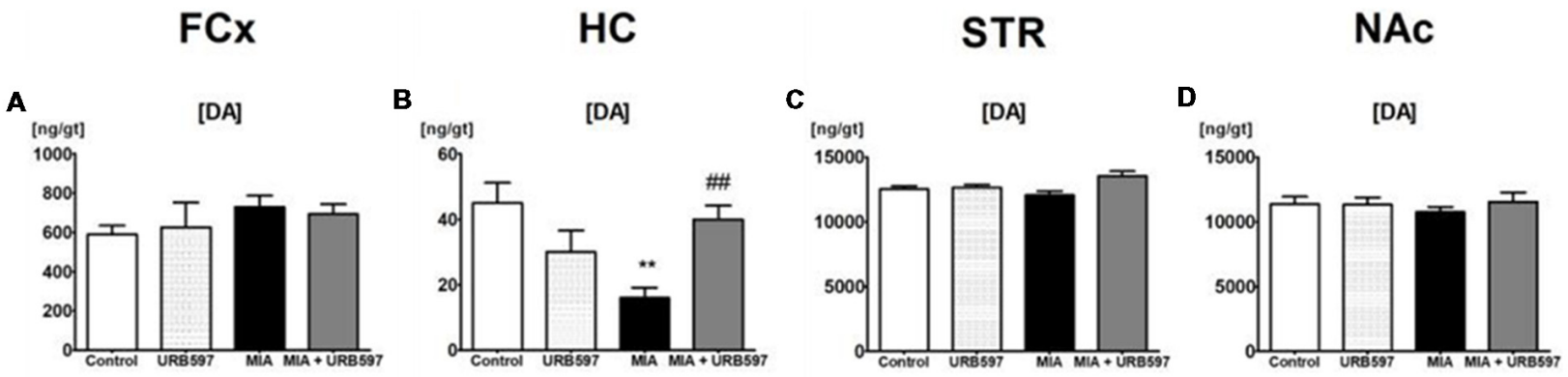

$\mathbf{E}$
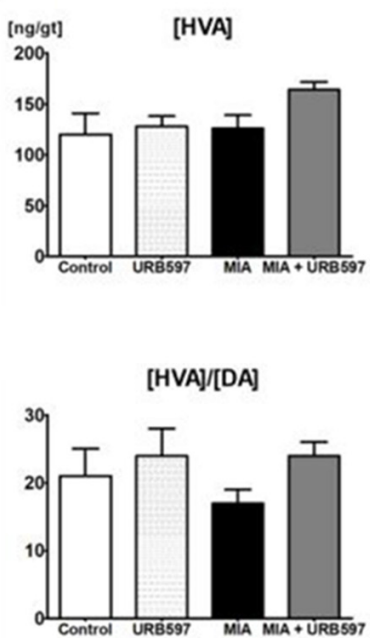

$\mathbf{F}$

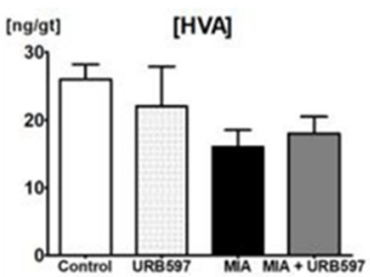

$\mathbf{J}$

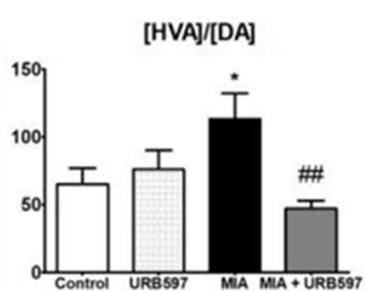

G

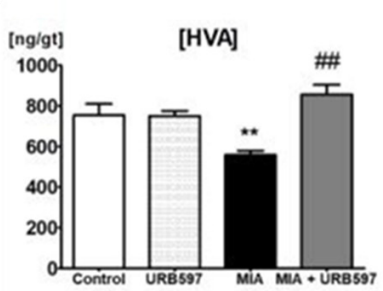

$\mathbf{K}$

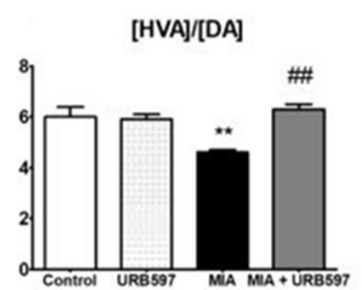

H

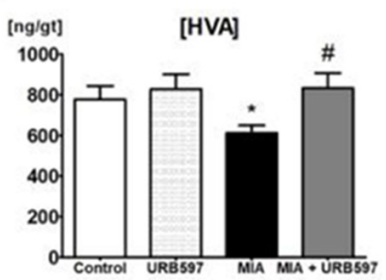

$\mathbf{L}$

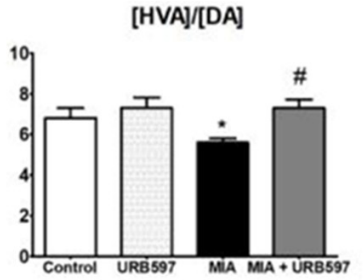

FIGURE 1 | The effect of URB597 on osteoarthritis (OA)-produced decreases in dopaminergic neurotransmission in the investigated brain structures; (A,E,I) frontal cortex (FCX), (B,F,J) hippocampus (HC), (C, G,K) striatum (STR) and (D,H,L) nucleus accumbens (NAc). Samples were collected 28 days after OA induction, $1 \mathrm{~h}$ after i.p. administration of URB597 or vehicle. Data are presented as the mean \pm SEM and represent normalized averages derived from 6 to 10 samples for each group. The results were analyzed by means of one-way analysis of variance (ANOVA), followed by Duncan's post hoc tests. Statistical significance: ${ }^{*} P<0.05$, ${ }^{* *} P<0.01$ vs. Control group; ${ }^{\#} P<0.05,{ }^{\#} P<0.01$ vs. OA group. One-way ANOVA indicated a significant effect of OA on the rate of dopamine (DA) metabolism in $\operatorname{STR}\left(F_{(3,22)}=13.89 ; P<0.00002\right)$ and NAc $\left(F_{(3,21)}=4.18 ; P<0.01\right)$; DA concentration in STR $\left(F_{(3,22)}=4.32 ; P<0.01\right)$ and $\mathrm{HC}\left(F_{(3,22)}=6.96 ; P<0.001\right)$ and homovanillic acid $(H V A)$ concentration in STR $\left(F_{(3,22)}=13.10 ; P<0.00004\right)$ and NAc $\left(F_{(3,21)}=3.48 ; P<0.03\right)$.

\section{RESULTS}

\section{The Effect of URB597 on OA-Produced Decreases in Dopaminergic Neurotransmission in the Brain}

\section{DA Concentrations}

One-way ANOVA showed significant effects of OA on DA concentrations in the STR and HC (Figures 1B,C and Supplementary Table S1) but no effects were observed in $\mathrm{FCx}$ and NAc (Figures 1A,D). Duncan's post hoc tests indicated that MIA significantly decreased the level of DA in the HC (approximately 60\%), and URB597 administration (1 mg/kg, i.p.) completely antagonized this effect and restored DA concentration to the control value (Figure 1B).

\section{HVA Concentrations (The Final Metabolite of DA)}

One-way ANOVA demonstrated a significant effect of OA on HVA concentrations in the STR and NAc (Supplementary
Table S1) but no effects were observed in FCx and HC (Figures 1E,F). Duncan's post hoc tests indicated that MIA significantly decreased HVA concentrations in the STR (approximately 30\%) and NAc (approximately 20\%). The same analysis showed that administration of URB597 completely antagonized the decreases in HVA concentration in the OA group (Figures 1G,H).

\section{Rates of DA Metabolism}

One-way ANOVA indicated a significant effect of OA on the rate of DA metabolism, presented as the ratio [HVA]/[DA] in the STR and NAc (Figures 1K,L and Supplementary Table S1) but no effects were observed in FCx and HC (Figures 1I,J). Duncan's post hoc tests revealed that OA significantly decreased the rate of DA metabolism in both investigated structures). URB597 significantly restored the rate of DA metabolism in both structures to the control values (Figures 1K,L). 

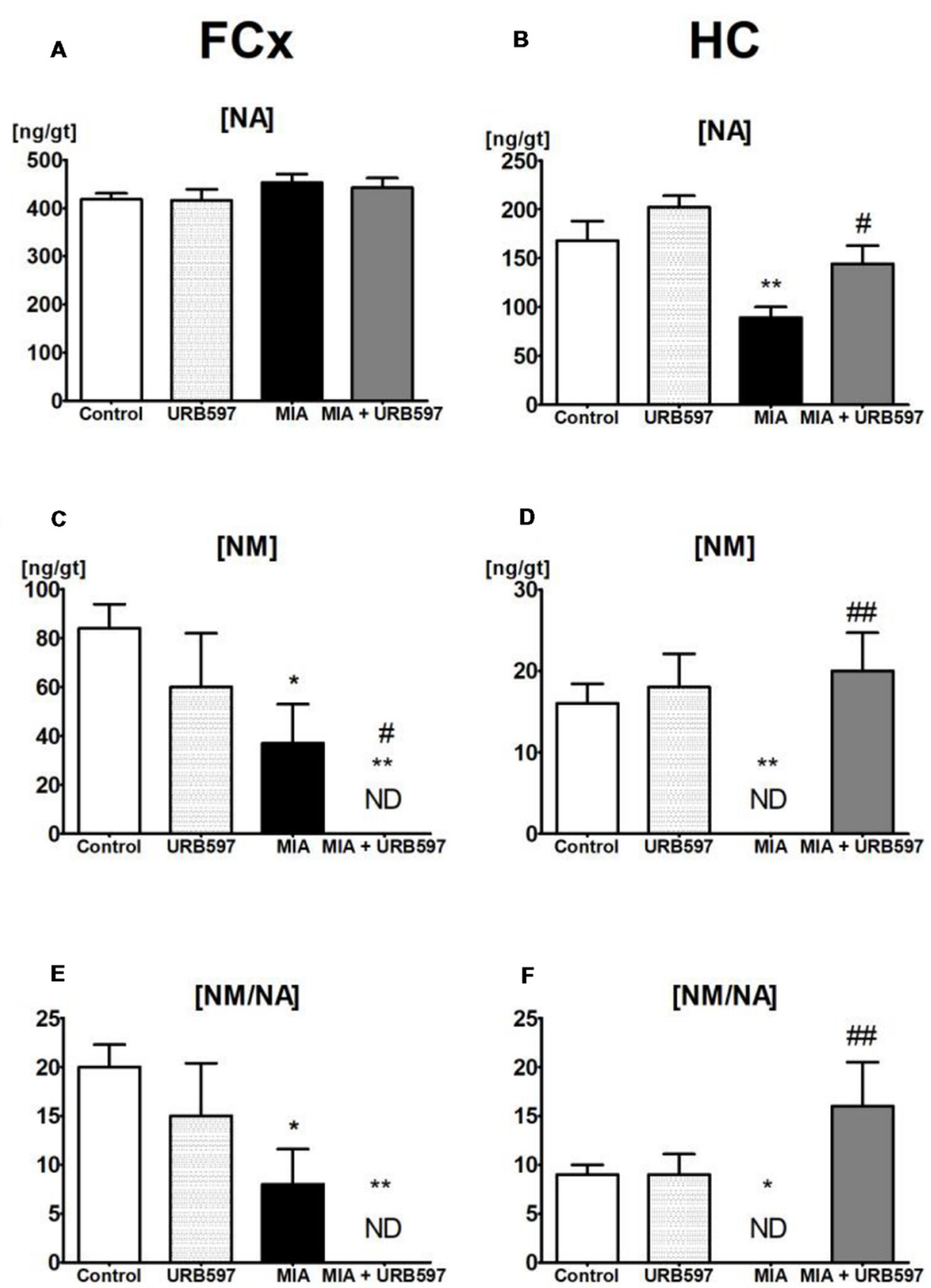

FIGURE 2 | The effect of URB597 on OA-produced decreases in noradrenergic neurotransmission in the investigated brain structures; (A,C,E) FCX and (B,D,F) HC. Samples were collected 28 days after OA induction, $1 \mathrm{~h}$ after i.p. administration of URB597 or vehicle. Data are presented as the mean \pm SEM and represent normalized averages derived from 6 to 10 samples for each group. The results were analyzed by means of one-way ANOVA, followed by Duncan's post hoc tests. Statistical significance: ${ }^{*} P<0.05,{ }^{* *} P<0.01$ vs. Control group; ${ }^{\#} P<0.05$, ${ }^{\# \#} P<0.01$ vs. OA group. One-way ANOVA indicated a significant effect of OA on the rate of noradrenaline (NA) metabolism in $\mathrm{FCx}\left(F_{(3,22)}=5.69 ; P<0.004\right)$ and $\mathrm{HC}\left(F_{(3,22)}=6.88 ; P<0.001\right)$; NM concentration in FCx $\left(F_{(3,22)}=5.66, P<0.004\right)$ and $\mathrm{HC}\left(F_{(3,22)}=8.92, P<0.0004\right)$ and NA concentration in the $\mathrm{HC}\left(F_{(3,22)}=9.99, P<0.0002\right)$.

\section{The Effect of URB597 on OA-Produced Changes in NA Transmission in the Brain}

\section{NA Concentrations}

One-way ANOVA showed a significant effect of OA on the NA concentration in the HC (Supplementary Table S2) and Duncan's post hoc test revealed a decrease in NA concentration (approximately 45\%) in the OA group (Figure 2B), however it was not affected in FCx (Figure 2A). URB597 administration to $\mathrm{OA}$ rats significantly antagonized the decrease in NA in the HC (Figure 2B).

\section{NM Concentrations (The Extraneuronal Metabolite of NA)}

ANOVA demonstrated a significant effect of OA on the concentrations of NM (Supplementary Table S2), which may 
be a good intermediate indicator of NA release in the $\mathrm{FCx}$ and HC. Duncan's post hoc tests showed that OA induced a strong decrease in NM concentrations in the HC (approximately $100 \%$ ) as well as in the FCx (approximately 60\%), and URB597 administration restored it to the control level in the $\mathrm{HC}$ but intensified the decrease in $\mathrm{NM}$ in the $\mathrm{FCx}$ (Figures 2C,D).

\section{Rates of NA Metabolism}

One-way ANOVA indicated a significant effect of OA on rates of NA metabolism, as shown by the ratio [NM]/[NA] in the FCx and HC (Supplementary Table S2). Duncan's post hoc tests revealed that $\mathrm{OA}$ significantly decreased rates of NA metabolism in the $\mathrm{FCx}$, and completely inhibited NA release in the HC. URB597 administration to OA-rats completely antagonized the decreased NA metabolism in the $\mathrm{HC}$ and significantly intensified the rate of NA metabolism in the FCX (Figures 2E,F).

\section{DISCUSSION}

Our results showed that the chronic pain state due to OA was reflected by significant inhibition of dopaminergic transmission. This result may reflect the high depression comorbidity observed in OA patients (Salamone et al., 2006, 2016). Furthermore, it is well known that dopaminergic transmission within NAc is an important factor in mediating the suppression of tonic pain (Altier and Stewart, 1999; Wood, 2008) and lesions within the mesostriatal pathway lead to the development of mechanical hypersensitivity (Takeda et al., 2005). URB597 treatment was able to reverse disruptions in dopaminergic signaling within the NAc in OA-rats; importantly, it had no effect on DA signaling in healthy rats. This not only reflects the antinociceptive potential of FAAH inhibitors (Jayamanne et al., 2006; Russo et al., 2007) but also suggests that an increase in EC tone may elicit rewarding and antidepressive effects, but only in impaired animals. Indeed, most recent findings have confirmed that inhibition of FAAH improved depressive-like behaviors induced by neuropathic pain. Interestingly, the peripherally restricted FAAH inhibitor failed to induce antidepressive effects despite retaining antinociceptive potential (Jiang et al., 2018). In addition, DA also exerts antinociceptive effects within the HC (Shamsizadeh et al., 2013; Reisi et al., 2014). Indeed, we also observed a decrease in DA levels in the $\mathrm{HC}$ following $\mathrm{OA}$ induction, which was reversed by URB597 treatment. In conclusion, URB597 is able to restore DA depletion due to OA-related chronic pain without affecting DA transmission in healthy animals.

Another significant factor in the top-down control of pain sensation is NA, which can exert opposite effects on pain transmission. For example, NA release mediates the inhibition of spinal nociceptive transmission by the descending pathways originating in the brainstem (Millan, 2002). Similar to DA, NA has been implicated as an antinociceptive neurochemical within the HC (Jin et al., 2014). In contrast, in chronic pain conditions, NA release in the FCx is actually responsible for pain generation (Taylor and Westlund, 2017). In our research, we observed a decline in NA release in both of these structures. URB597 was able to reverse NA levels in the HC, however it also caused a complete depletion of NA release in the FCx, without affecting NA concentration. These results are probably related to the analgesic potential of URB597.

In summary, our results demonstrated that increasing EC tone, counteracts the hypodopaminergic state in $\mathrm{OA}$ animals without affecting healthy animals. However, these results are not in line with the clinical research that has shown a lack of efficacy of FAAH inhibitors in reducing pain in OA (Huggins et al., 2012) and symptoms of depression (Sanofi, 2013). It is not known what causes this discrepancy between the very promising preclinical data and the lack of FAAH inhibitor efficacy in the clinic. Off-target activity of AEA or URB597 itself could play an important role. For example, AEA is also a TRPV1 agonist (Starowicz et al., 2013) and thus it may mediate pro-nociceptive effects as well. Additionally, the biological actions of FAAH extend far beyond the termination of anandamide signaling. Broad changes in lipid levels may arise as a result of the redundancy of EC inactivation (Piscitelli and Di Marzo, 2012). Moreover, in light of the present study it is important to note poor specificity of URB597 (Zhang et al., 2007), which has been shown to upregulate tyrosine hydrolase independently FAAH inhibition (Bosier et al., 2013). This study was preliminary and therefore, the exact mechanism of action was not established and off-target activity of URB597 may not be excluded. Further experiments with use of more specific FAAH inhibitors and cannabinoid receptor antagonists are required.

\section{DATA AVAILABILITY}

The raw data supporting the conclusions of this manuscript will be made available by the authors, without undue reservation, to any qualified researcher.

\section{AUTHOR CONTRIBUTIONS}

JM was responsible for drug treatment, participated in the study design, data interpretation and preparation of manuscript. AW performed the experiment and collected the data. JTM conducted the statistical analyses. LA-M participated in study design, data interpretation and preparation of manuscript. KS conceived and designed the study and was involved in interpretation of the data, preparation of the manuscript and revisions for intellectual content.

\section{FUNDING}

This work was supported by Departmental Statutory funds (Institute of Pharmacology, Polish Academy of Sciences). 


\section{ACKNOWLEDGMENTS}

We gratefully acknowledge Irena Romańska, M.Sc. for professional assistance with the sample dissections and neurochemical studies.

\section{REFERENCES}

Altier, N., and Stewart, J. (1999). The role of dopamine in the nucleus accumbens in analgesia. Life Sci. 65, 2269-2287. doi: 10.1016/s0024-3205(99) 00298-2

Antkiewicz-Michaluk, L., Michaluk, J., Mokrosz, M., Romanska, I., LorencKoci, E., Ohta, S., et al. (2001). Different action on dopamine catabolic pathways of two endogenous 1,2,3,4-tetrahydroisoquinolines with similar antidopaminergic properties. J. Neurochem. 78, 100-108. doi: 10.1046/j.14714159.2001.00391.x

Apkarian, A. V., Baliki, M. N., and Farmer, M. A. (2013). Predicting transition to chronic pain. Curr. Opin. Neurol. 26, 360-367. doi: 10.1097/WCO. 0b013e32836336ad

Baliki, M. N., Petre, B., Torbey, S., Herrmann, K. M., Huang, L., Schnitzer, T. J., et al. (2012). Corticostriatal functional connectivity predicts transition to chronic back pain. Nat. Neurosci. 15, 1117-1119. doi: 10.3410/f.717952426. 793462973

Bosier, B., Muccioli, G. G., and Lambert, D. M. (2013). The FAAH inhibitor URB597 efficiently reduces tyrosine hydroxylase expression through CBand FAAH-independent mechanisms. Br. J. Pharmacol. 169, 794-807. doi: 10.1111/j.1476-5381.2012.02208.x

Hassett, A. L., Aquino, J. K., and Ilgen, M. A. (2014). The risk of suicide mortality in chronic pain patients. Curr. Pain Headache Rep. 18:436. doi: 10.1007/s11916014-0436-1

Huggins, J. P., Smart, T. S., Langman, S., Taylor, L., and Young, T. (2012). An efficient randomised, placebo-controlled clinical trial with the irreversible fatty acid amide hydrolase-1 inhibitor PF-04457845, which modulates endocannabinoids but fails to induce effective analgesia in patients with pain due to osteoarthritis of the knee. Pain 153, 1837-1846. doi: 10.1016/j.pain.2012. 04.020

International Association for the Study of Pain (IASP). (2018). IASP Guidelines for the Use of Animals in Research. Available online at: http://www.iasppain.org/Education/Content.aspx?ItemNumber=1217 [Accessed September $25,2018]$

Jayamanne, A., Greenwood, R., Mitchell, V. A., Aslan, S., Piomelli, D., and Vaughan, C. W. (2006). Actions of the FAAH inhibitor URB597 in neuropathic and inflammatory chronic pain models. Br. J. Pharmacol. 147, 281-288. doi: 10.1038/sj.bjp.0706510

Jiang, H.-X., Ke, B.-W., Liu, J., Ma, G., Hai, K.-R., Gong, D.-Y., et al. (2018). Inhibition of fatty acid amide hydrolase improves depressive-like behaviors independent of its peripheral antinociceptive effects in a rat model of neuropathic pain. Anesth. Analg. doi: 10.1213/ane.0000000000003563 [Epub ahead of print].

Jin, H., Teng, Y., Zhang, X., Yang, C., Xu, M., and Yang, L. (2014). Noradrenergic mechanism involved in the nociceptive modulation of hippocampal CA3 region of normal rats. Neurosci. Lett. 574, 31-35. doi: 10.1016/j.neulet.2014.05.012

Leite, A. A., Costa, A. J. G., de Lima, B. A. M., Padilha, A. V. L., and de Albuquerque, E. C., and Marques, C. D. L. (2011). Comorbidades em pacientes com osteoartrite: frequência e impacto na dor e na função física. Rev. Bras. Reumatol. 51, 118-123. doi: 10.1590/S0482-50042011000200002

Leuchter, A. F., Husain, M. M., Cook, I. A., Trivedi, M. H., Wisniewski, S. R., Gilmer, W. S., et al. (2010). Painful physical symptoms and treatment outcome in major depressive disorder: a STAR*D (Sequenced Treatment Alternatives to Relieve Depression) report. Psychol. Med. 40, 239-251. doi: 10.1017/s0033291709006035

Mansour, A. R., Baliki, M. N., Huang, L., Torbey, S., Herrmann, K. M., Schnitzer, T. J., et al. (2013). Brain white matter structural properties predict transition to chronic pain. Pain 154, 2160-2168. doi: 10.1016/j.pain.2013. 06.044

\section{SUPPLEMENTARY MATERIAL}

The Supplementary Material for this article can be found online at: https://www.frontiersin.org/articles/10.3389/fnmol.2018.004 66/full\#supplementary-material

Martikainen, I. K., Nuechterlein, E. B., Peciña, M., Love, T. M., Cummiford, C. M. Green, C. R., et al. (2015). Chronic back pain is associated with alterations in dopamine neurotransmission in the ventral striatum. J. Neurosci. 35, 9957-9965. doi: 10.1523/jneurosci.4605-14.2015

Millan, M. J. (2002). Descending control of pain. Prog. Neurobiol. 66, 355-474. doi: 10.1016/S0301-0082(02)00009-6

Nazarinasab, M., Motamedfar, A., and Moqadam, A. E. (2017). Investigating mental health in patients with osteoarthritis and its relationship with some clinical and demographic factors. Reumatologia 4, 183-188. doi: 10.5114/reum. 2017.69778

Nebe, A., and Ebersbach, G. (2009). Pain intensity on and off levodopa in patients with Parkinson's disease. Mov. Disord. 24, 1233-1237. doi: 10.1002/mds.22546

Piscitelli, F., and Di Marzo, V. (2012). "Redundancy" of endocannabinoid inactivation: new challenges and opportunities for pain control. ACS Chem Neurosci. 3, 356-363. doi: 10.1021/cn300015x

Reisi, Z., Haghparast, A., Pahlevani, P., Shamsizadeh, A., and Haghparast, A. (2014). Interaction between the dopaminergic and opioidergic systems in dorsal hippocampus in modulation of formalin-induced orofacial pain in rats. Pharmacol. Biochem. Behav. 124, 220-225. doi: 10.1016/j.pbb.2014.06.015

Russo, R., LoVerme, J., La Rana, G., Compton, T. R., Parrott, J., Duranti, A., et al (2007). The fatty acid amide hydrolase inhibitor URB597 (cyclohexylcarbamic acid 3'-carbamoylbiphenyl-3-ylester) reduces neuropathic pain after oral administration in mice. J. Pharmacol. Exp. Ther. 322, 236-242. doi: 10.1124/jpet.107.119941

Salamone, J. D., Yohn, S. E., López-Cruz, L., San Miguel, N., and Correa, M. (2016) Activational and effort-related aspects of motivation: neural mechanisms and implications for psychopathology. Brain 139, 1325-1347. doi: 10.1093/brain/ aww050

Salamone, J., Correa, M., Mingote, S., Weber, S., and Farrar, A. (2006). Nucleus accumbens dopamine and the forebrain circuitry involved in behavioral activation and effort-related decision making: implications for understanding anergia and psychomotor slowing in depression. Curr. Psychiatry Rev. 2, 267-280. doi: 10.2174/157340006776875914

Sanofi (2013). An eight-week, multicenter, randomized, double-blind, placebocontrolled dose-finding study, with escitalopram (10 mg daily) as active control, to evaluate the efficacy, safety and tolerability of three fixed doses of SSR411298 (10,50, or $200 \mathrm{mg}$ daily) in elderly patients with major depressive disorder. Available online at: https://www.clinicaltrialsregister.eu/ctrsearch/rest/download/result/attachment/2008-001718-26/1/8303

Shamsizadeh, A., Pahlevani, P., Haghparast, A., Moslehi, M., Zarepour, L., and Haghparast, A. (2013). Involvement of dopamine receptors within the dorsal hippocampus in suppression of the formalin-induced orofacial pain Pharmacol. Biochem. Behav. 114-115, 37-42. doi: 10.1016/j.pbb.2013.10.029

Skogar, O., and Lökk, J. (2016). Pain management in patients with Parkinson's disease: challenges and solutions. J. Multidiscip. Healthc. 9, 469-479. doi: 10.2147/JMDH.S105857

Starowicz, K., Makuch, W., Korostynski, M., Malek, N., Slezak, M., Zychowska, M., et al. (2013). Full inhibition of spinal FAAH leads to TRPV1-mediated analgesic effects in neuropathic rats and possible lipoxygenase-mediated remodeling of anandamide metabolism. PLoS One 8:e60040. doi: 10.1371/journal.pone. 0060040

Takeda, R., Ikeda, T., Tsuda, F., Abe, H., Hashiguchi, H., Ishida, Y., et al. (2005) Unilateral lesions of mesostriatal dopaminergic pathway alters the withdrawal response of the rat hindpaw to mechanical stimulation. Neurosci. Res. 52, 31-36. doi: 10.1016/j.neures.2005.01.005

Taylor, B. K., and Westlund, K. N. (2017). The noradrenergic locus coeruleus as a chronic pain generator. J. Neurosci. Res. 95, 1336-1346. doi: 10.1002/jnr.23956

Vachon-Presseau, E., Tétreault, P., Petre, B., Huang, L., Berger, S. E., Torbey, S., et al. (2016). Corticolimbic anatomical characteristics predetermine risk for chronic pain. Brain 139, 1958-1970. doi: 10.1093/brain/aww100 
Wood, P. B. (2008). Role of central dopamine in pain and analgesia. Expert Rev. Neurother. 8, 781-797. doi: 10.1586/14737175.8.5.781

Young Blood, M. R., Ferro, M. M., Munhoz, R. P., Teive, H. A. G., and Camargo, C. H. F. (2016). Classification and characteristics of pain associated with Parkinson's disease. Parkinsons. Dis. 2016, 1-8. doi: 10.1155/2016/6067132

Zhang, D., Saraf, A., Kolasa, T., Bhatia, P., Zheng, G. Z., Patel, M., et al. (2007). Fatty acid amide hydrolase inhibitors display broad selectivity and inhibit multiple carboxylesterases as off-targets. Neuropharmacology 52, 1095-1105. doi: 10.1016/j.neuropharm.2006. 11.009
Conflict of Interest Statement: The authors declare that the research was conducted in the absence of any commercial or financial relationships that could be construed as a potential conflict of interest.

Copyright (c) 2018 Mlost, Wasik, Michaluk, Antkiewicz-Michaluk and Starowicz. This is an open-access article distributed under the terms of the Creative Commons Attribution License (CC BY). The use, distribution or reproduction in other forums is permitted, provided the original author(s) and the copyright owner(s) are credited and that the original publication in this journal is cited, in accordance with accepted academic practice. No use, distribution or reproduction is permitted which does not comply with these terms. 